\title{
Representation of a function in terms of its mean boundary values
}

\section{Chin-Hung Ching and Charles K. Chui}

\begin{abstract}
We give the representation formula of a function in a disc in terms of its arithmetic means on the boundary. We also give applications of this formula to the solutions of some elementary differential equations with prescribed means of boundary or initial data.
\end{abstract}

Let $U$ denote the open unit disc and $T$ the unit circle. For a function $f$ continuous on $T$, we consider its arithmetic means

$$
s_{n}(f)=\frac{1}{n} \sum_{k=1}^{n} f\left(e^{i 2 \pi k / n}\right),
$$

$n=1,2, \ldots$ and

$$
s_{\infty}(f)=\lim _{n \rightarrow \infty} s_{n}(f)
$$

In $[1,2]$, it is noted that if $f$ is "smooth", the sequence

$$
r_{n}(f)=s_{n}(f)-s_{\infty}(f)
$$

has the same rate of convergence to zero as the Fourier coefficients $a_{n}(f)$ of $f$. We call $r_{n}(f)$ the $n$th Riemann coefficient of $f$, and denote $r_{0}(f)=s_{\infty}(f)$. Let $\mu(n)$ be the Möbius function and consider the polynomials $p_{0}(z)=1$ and

Received 27 June 1972. 


$$
p_{n}(z)=\sum_{s\lceil n} \mu\left(\frac{n}{s}\right) z^{s},
$$

$n=1,2, \ldots$. We announce the following result.

THEOREM. Let $f \in C^{\mathcal{l}+\varepsilon}(T)$ for some $\varepsilon>0$ and let $r_{n}(f)$ be the Riemonn coefficients of $f$. Then the series

$$
\sum_{n=0}^{\infty} r_{n}(f) p_{n}(z)
$$

converges uniformly on the closed unit dise $\bar{U}$ to a unique function $F$. Furthermore, if $f$ admits an analytic continuation in $U$, then $F=f$ on $T$.

We call (1) the Riemann series of $f$. It should be remarked that in the proof of the above theorem, we need only assume that the Fourier coefficients $a_{n}(f)$, or equivalently the Riemann coefficients $r_{n}(f)$, are of order $O\left(1 / n^{1+\varepsilon}\right)$. Also, there is a sequence $r_{n}=O(1 / n)$ such that the series $\sum r_{n} p_{n}$ diverges for all $z \neq 0$. As an application, we give some existence and uniqueness theorems of the solutions of some elementary differential equations with prescribed arithmetic means of boundary or initial values.

(1) Let $\left\{\alpha_{n}\right\},\left\{\beta_{n}\right\},\left\{\gamma_{n}\right\}$ and $\left\{\delta_{n}\right\}$ be sequences of real numbers converging to $\alpha, 0,0$ and 0 respectively with the rates $\alpha_{n}-\alpha=O\left(1 / n^{3+\varepsilon}\right), B_{n}=O\left(1 / n^{2+\varepsilon}\right), \gamma_{n}=O\left(1 / n^{2+\varepsilon}\right)$ and $\delta_{n}=O\left(1 / n^{1+\varepsilon}\right)$ where $\varepsilon>0$. Then there exists a unique function $u(x, t)$ in $c^{2+\varepsilon^{\prime}}\left(R^{2}\right)$ for some $\varepsilon^{\prime}>0$ such that

$$
\begin{array}{r}
\frac{\partial^{2} u}{\partial t^{2}}=\frac{\partial^{2} u}{\partial x^{2}}, \\
\frac{1}{n} \sum_{k=1}^{n} u\left(j+\frac{k}{n}, 0\right)=\alpha_{n}, \\
\frac{1}{n} \sum_{k=1}^{n} u_{x}\left(j+\frac{k}{n}, 0\right)=\beta_{n},
\end{array}
$$




$$
\begin{aligned}
& \frac{1}{n} \sum_{k=1}^{n} u_{t}\left(j+\frac{k}{n}, 0\right)=\gamma_{n}, \\
& \frac{1}{n} \sum_{k=1}^{n} u_{t x}\left(j+\frac{k}{n}, 0\right)=\delta_{n}
\end{aligned}
$$

for all $j=0, \pm 1, \ldots$ and $n=1,2, \ldots$.

(II) Let $\left\{\alpha_{n}\right\}$ and $\left\{\beta_{n}\right\}$ be sequences of real numbers converging to $\alpha$ and 0 respectively with the rates $\alpha_{n}-\alpha=O\left(1 / n^{3+\varepsilon}\right)$ and $\beta_{n}=O\left(1 / n^{2+\varepsilon}\right)$ for some $\varepsilon>0$. Then there exists a unique function $u(r, \theta)$ in $C^{2+\varepsilon^{\prime}}(U)$ for some $\varepsilon^{\prime}>0$ such that

$$
\begin{aligned}
\Delta u & =0 \text { in } U, \\
\frac{1}{n} \sum_{k=1}^{n} u\left(1, \frac{2 \pi k}{n}\right) & =\alpha_{n}, \\
\frac{1}{n} \cdot \sum_{k=1}^{n} u_{\theta}\left(1, \frac{2 \pi k}{n}\right) & =\beta_{n},
\end{aligned}
$$

for all $n=1,2, \ldots$.

By similar methods, we can obtain some analogous results about the solutions for the heat equation with prescribed mean initial values and also for the Laplace equation with prescribed means of the normal derivatives. The details of the proof of the theorem and other related results will appear elsewhere.

\section{References}

[1] Chin-Hung Ching and Charles K. Chul, "Asymptotic similarities of Fourier and Riemann coefficients", J. Approximation Theory (to appear).

[2] Charles K. Chui, "Concerning rates of convergence of Rlemann sums", $J$. Approximation Theory 4 (1971), 279-287.

Texas A \& M University,

Texas, USA. 